\title{
Intradimensional vs. extradimensional transfer in the discriminative learning of goldfish and pigeons
}

\author{
PATRICIA A. COUVILLON, W. A. TENNANT, and M. E. BITTERMAN \\ University of Hawaii, Honolulu, Hawaii 96822
}

\begin{abstract}
Evidence of better intradimensional than extradimensional transfer was sought in naive goldfish trained under free-operant single-stimulus conditions (Experiment I), sophisticated pigeons trained under free-operant single-stimulus conditions (Experiment II), sophisticated pigeons trained under discrete-trials choice conditions (Experiment III), and naive pigeons trained under discretetrials choice conditions (Experiment IV). The results provide no support for attention theory.
\end{abstract}

Greater intradimensional (ID) than extradimensional (ED) transfer has been found in experiments with rats (Shepp \& Eimas, 1964), pigeons (Mackintosh \& Little, 1969), and monkeys (Shepp \& Schrier, 1969); that is, discriminative learning in these animals has been reported to be more rapid when the relevant dimension of a preceding problem remains relevant than when a previously irrelevant dimension becomes relevant. These results have been interpreted in attentional terms and, in fact, constitute some of the strongest evidence for attention theory (Sutherland \& Mackintosh, 1971). Analogous results have failed, however, to appear either in goldfish (Tennant \& Bitterman, 1973) or in carp (Tennant \& Bitterman, 1975), and our aim here has been to try to understand the discrepancy. In our experiments with fishes, unlike those with the other animals, sophisticated rather than naive subjects were studied and a free-operant singlestimulus method rather than a discrete-trials choice method was employed. There is, of course, nothing in attentional theory to suggest that the dimensional transfer effect should be found only in naive animals or only when a choice method is used, and the discovery of such restrictions would make some difficulty for the theory.

\section{EXPERIMENT I}

In this experiment, we looked for the dimensional transfer effect in naive goldfish under free-operant conditions. The animals were trained concurrently with two sets of stimuli varying in the same two dimensions (color and orientation of a line). The same dimension was relevant in both sets of stimuli for the ID group, while for the ED group the relevant dimension of one set was the irrelevant

This work was supported by Grant MH-23294 from the Public Health Service. Reprints may be obtained from M. E. Bitterman, Laboratory of Sensory Sciences, 1993 East-West Road, Honolulu, Hawaii 96822 . dimension of the other. It will be noted, of course, that we have here yet another departure from the conventional ID-ED experimental design in that the "training" and "transfer" problems are encountered concurrently. In fact, each problem provides a measure of transfer, because the facilitating effect of dimensional congruence predicted from attention theory should be evident in both, as should also the predicted retarding effect of dimensional incongruence. If experience with one set of stimuli differentially strengthens attention in one of the two dimensions, response to the other set should be influenced accordingly. Concurrent discrimination in the two sets by ED animals actually cannot be accounted for in attentional terms unless it is assumed that the animals can somehow "condition the strength of a given analyzer to outputs from other analyzers" (Sutherland \& Mackintosh, 1971, p. 163). Despite the disarming simplicity of its central concepts, attention theory is quite inadequate to the facts of discriminative learning without a substantial array of such supporting assumptions (Bitterman, 1972). In any case, we hoped here that, with the more powerful concurrent design, we should be less likely to miss any small dimensional transfer effect which might exist.

\footnotetext{
Method

Subjects. The subjects were 12 experimentally narve $10-\mathrm{cm}$ goldfish. They were maintained on a $24-\mathrm{h}$ feeding schedule in a temperature-controlled laboratory room.

Apparatus. The 12 individual living tanks, their two long sides and back ends painted flat black, were arranged on a circular table which could be rotated to bring each tank in turn into the training position. The training apparatus, mounted on a chasis of black Plexiglas, covered the top and front end of the tank, providing a darkened experimental enclosure. The manipulandum was a circular target, $4 \mathrm{~cm}$ in diameter, with a feeding nipple at its center through which liquid food could be delivered (Woodard \& Bitterman, 1974). It was situated at the front end of the tank behind a circular opening in a black Plexiglas surround designed to require the fish to approach the target on a perpendicular course. The target could be illuminated from behind, either diffusely by a white lamp, or by an optical system designed to project a line
} 
of light $(3.2 \mathrm{~cm}$ long and $1.5 \mathrm{~mm}$ wide, the reedng mpple at its centerl on a dark background The line of light, which bisected the tesponse key, could be varied in color and in orientation The colors used in this experiment were red (Wratten No. 25), vellow (three thicknesses of No 8), green (No. 58), and hlue (No 47); the light source was a $200-\mathrm{W}$ Sylvania projector lamp (CGW) operated at $65 \mathrm{~V}$. The orientations of the line were $0^{\circ}$ (horizontal), $45^{\circ}, 90^{\circ}$ (vertical), and $135^{\circ}$. The trainıng apparatus was cabled to programming equipment in an adjacent room All events of the experiment, including changes in color and onentation of the line, were controlled automatically, and responses were recorded with a printing counter.

Procedure. The animals were pretrained to strike at a white target on a schedule of continuous reinforcement followed by variable interval (VI) schedules of $10 \mathrm{sec}, 15 \mathrm{sec}, 30 \mathrm{sec}$, and $1 \mathrm{~min}$. The procedure employed in the experiment proper was to present the stımulı to be discriminated for 3-min periods. The presentations were separated by intervals in darkness of $5 \mathrm{sec}$ or longer (Interval responding was penalized by resetting of the interval clock). Responses during presentations of a positive stimulus were reinforced with drops of food on a VI 1 -min schedule, while responses during presentations of a negative stimulus were unreinforced. The measure of performance was the number of responses made during each presentation exclusive of the number of responses made in the 5 -sec period following each scheduled reinforcement, the purpose of this exclusion being to eliminate consummatory responses from the measure of performance. The program was the same on unreinforced as on reinforced presentations except that no food actually was delivered. Each session began with a warm-up period, during which the white target was presented and response to it reinforced on the VI 1-min schedule until the animal had made at least 40 responses in $3 \mathrm{~min}$.

All animals were trained concurrently with two sets of four stimuli each-one set consisting of red and yellow horizontals and verticals, and a second consisting of blue and green diagonals. There were two presentations of each of the eight stimuli in balanced orders in each of 24 sessions. For six anımals comprising the ID group, the same dimension was relevant in both sets of stimuli; for three of the animals, color was relevant and orientation irrelevant, while orientation was relevant and color irrelevant for the remaining three. Six animals comprising the ED group were trained with different relevant dimensions in the two sets of stimuli; for three of the anmals, color was relevant in the red-yellow set and orientation relevant in the blue-green set, while the reverse was true for the other three animals. In Table 1, the procedure is illustrated in terms of the treatment of four sample animals.

Table 1

Design of Experiment I Illustrated in Terms of Treatment of Four Sample Subjects

\begin{tabular}{ccccc}
\hline Stimuli & 1 & 2 & 3 & 4 \\
\hline R, V & + & + & + & + \\
R, H & + & - & + & - \\
Y, V & - & + & - & + \\
Y, H & - & - & - & - \\
G, Da & + & + & + & + \\
G, Db & + & + & - & - \\
B, Da & - & - & + & + \\
B, Db & - & - & - & - \\
\hline
\end{tabular}

Note-Abbreviations: $R=$ red, $Y=$ yellow, $G=$ green, $B=$ blue, $V=$ vertical, $H=$ horizontal, $D a$ and $D b=$ diagonals, $+=$ reinforced, - = unreinforced. Subjects 1 (color relevant in both sets of stimuli) and 4 (orientation relevant in both sets of stimuli) are ID antmals, while Subjects 2 (orientation, color) and 3 (color, orientation) are ED animals.

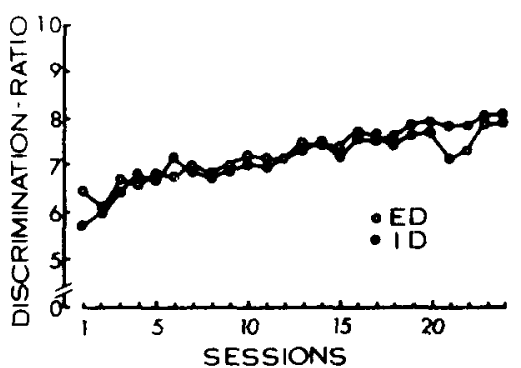

Figure 1. The performance of naive goldfish under freeoperant conditions in Experiment I. ED, extradimensional; ID, intradimensional.

\section{Results}

As Figure 1 shows, the discriminative performance of the ID and ED animals was virtually identical (performance was expressed in terms of the conventional discrimination ratio), and analysis of variance failed to yield any indication either of a groups effect or of an interaction of Groups by Sessions $(F<1)$. The performance of both groups was rather poor relative to that in a previous experiment with sophisticated goldfish (Tennant \& Bitterman, 1973), perhaps because of the inexperience of the present (naive) subjects. Whatever the reason, there should have been ample opportunity here to see a facilitating effect of dimensional congruence, or a retarding effect of dimensional incongruence, or both, but it made no difference at all whether the same dimension was relevant in both sets of stimuli or whether the relevant dimension of one set was the irrelevant dimension of the other. There is no evidence, then, of the dimensional transfer effect in naive goldfish trained under free-operant conditions.

\section{EXPERIMENT II}

We looked now for the dimensional transfer effect in a free-operant experiment with experienced pigeons. If experienced pigeons showed the effect under conditions like those under which experienced goldfish had failed to show it in the original experiment (Tennant \& Bitterman, 1973), a phyletic difference would be indicated. It may be well to emphasize perhaps that according to attention theory the specific effects of prior training should be reversible. That is, differential reinforcement in a subsequent two-dimensional problem with one dimension relevant and the other irrelevant must ultimately strengthen attention in the relevant dimension and weaken it in the irrelevant dimension-the animal cannot master the new problem until that happensand the difference in strength should influence response to any other set of stimuli varying in the same two dimensions. 


\section{Method}

Subjects. The subjects were 12 male homing pigeons obtained from a local breeder and mantained at $80 \%$ of free-feeding weight in a ventilated and temperature-controlled laboratory room Of the 12 anımals, 4 had previously been used in an experiment on probability learning and 8 in an experiment on reversal learning. All had had experience with a considerable variety of stimuli.

Apparatus. The subjects were trained in a ventilated picnic chest set into a larger, sound-attenuated enclosure Centered on one wall of the inner compartment was a single pecking key which could be illuminated by an Industrial Electronıcs one-plane readout projector. The stimuli (all projected on a dark ground) were a white circle and a series of straight lines ( $2.5 \mathrm{~cm}$ long and $0.25 \mathrm{~cm}$ wide), varying both in color and in orientation. which bisected the kev. The colors were red (Wratten No. 25), yellow ( 9 thicknesses of No. 8), blue (No. 47), and green ( 3 thicknesses of No. 58). The orientations were $0^{\circ}$ (horizontal) $45^{\circ}, 90^{\circ}$ (vertical), and $135^{\circ}$. Directly below the key was a feeder-a motor-driven box filled with mixed grain. Normally retracted from the animal's compartment, the box could be introduced into the compartment for each scheduled 2.5 -sec reinforcement period, during which it was illuminated with a white light. All events of the experiment were controlled automatıcally, and the responses were recorded with a printing counter.

Procedure. The anımals were adapted to the experimental situation in eight sessions of VI 1-min tranng with the white circle. In each daily session, there were $103-\mathrm{mm}$ presentations of the white circle separated by 5 -sec intervals in the dark. durnng which pecking was unreinforced. To acquaint the animals with the sumuls to be used in the experiment, two additional sessions were given in which each of the eight stimuli-the red and yellow horizontals and verticals, and the blue and green diagonals - was presented once and reinforced on the VI 1-min schedule. Here again, as in all subsequent training, the stımulus presentations were $3 \mathrm{~mm}$ in length and separated by 5 -sec intervals in darkness.

In Stage 1 of the experiment, the anımals were trained for 15 sessions with the red and yellow horizontals and verticals. For six of the animals, color was relevant and orientation irrelevant; red was positive for three and yellow for three. For the remainıng animals, orientation was relevant and color irrelevant; horizontal was positive for three and vertical for three. The stumuli were programmed in balanced orders, three presentations of each stimulus in each session. In Sessions 16-21, the animals were trained with blue and green diagonals, and, as before, there were three presentations of each stimulus in each session. For one yellow-positıve, two red-positive, one vertical-positive, and two horizontal-positıve animals, color was relevant and orientation irrelevant (green positive for three and blue positive for three). For the remaining animals, orientation was relevant and color irrelevant $\left(45^{\circ}\right.$ positive for three and $135^{\circ}$ positive for three). Thus, for half the animals (the ID group), the same dimension was relevant in both problems (color for three and orientation for three), while for the remaining animals (the ED group) the relevant dimension of the first problem was irrelevant in the second. In Session 22 , the animals were tested with all eight stimuli-two presentations of each--in balanced orders and reinforced as before. The prediction from attention theory here, of course, is that concurrent training with the two sets of stimuli should disrupt at least temporarily the performance of the ED animals.

In Stage 2, the effect of reversal was studied. There were 10 sessions with all eight stımuli--two presentations of each stimulus in each session in balanced orders. The stimuli in the red-yellow set were differentially reinforced as before, but positive and negawe stimul in the blue-green set were reversed, green rather than blue, or blue rather than green. being reinforced for the color animals, and $45^{\circ}$ rather than $135^{\circ}$, or $135^{\circ}$ rather than $45^{\circ}$. being reinforced for the orientation animals. The prediction from attention theory here is that (since speed of reversal is assumed to increase with strength of attention in the relevant dimension) the ID animals should reverse more rapidly.

In Stage 3, the effect of a dimensional shift was studied. There were 11 sessions with all enght stimul, two presentations of each stimulus in each session. Stimuli of the blue-green set were differentially reinforced as in the preceding stage, but the relevant and irrelevant dimensions were interchanged for the red-yellow set. One red-positıve and two yellow-positive animals were shifted to horizontal-positive, while the remaining colorrelevant animals were shifted to vertical-positive. One verticalpositive and two horizontal-positive animals were shifted to redpositive, while the remaining orientation-relevant animals were shifted to yellow-positive. With these changes, the previous ID anımals became ED animals (the relevant dimcnsicn of one set of stimuli now was the irrelevant dimension of the other set), while the previous ED animals became ID animals (same dimension relevant in both sets). The prediction from attention theory here is that the new ED animals should find the shift difficult because they come to the problem with stronger attention in the now irrelevant dimension and because that dimension continues to be relevant in the other set of stimuli. By the same logic, the new ID animals should find the shift much less difficult.

\section{Results}

The performance of the two groups in the three stages of the experiment is plotted in Figure 2. That the two groups were well matched at the outset is indicated by the data for the first 15 sessions of Stage 1, in which the animals were trained with the red-yellow horizontals and verticals. Subsequent

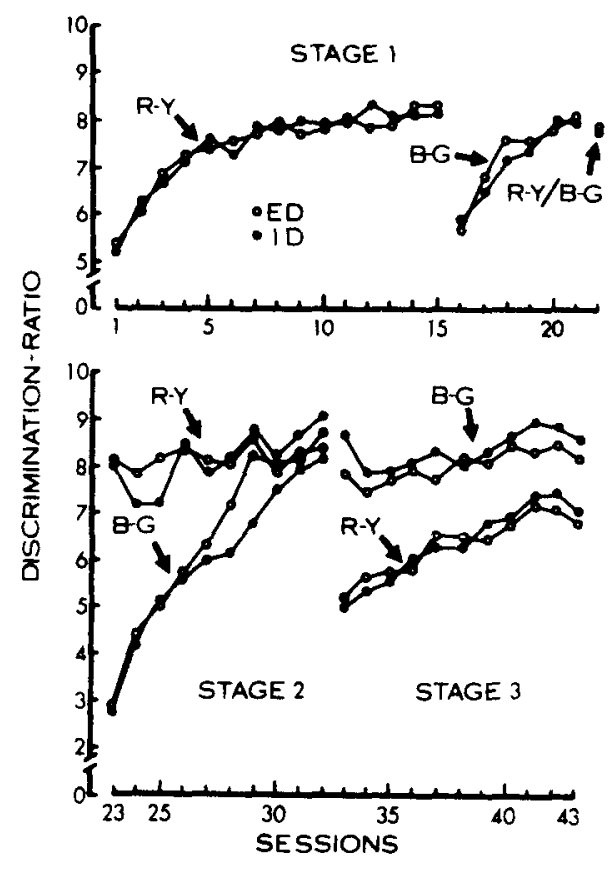

Figure 2. The performance of sophisticated pigeons under free-operant conditions in Experiment II. ED, extradimensional; ID, intradimensional; R-Y, red and yellow horizontals and verticals; B-G, blue and green diagonals; R-Y/B-G, both sets of stimuli combined. 
performance with the blue-green set was better, if anything, in the ED group, although the difference is statistically insignificant $(F<1)$, and the ED group was no less able than the ID group to deal with both sets of stimuli concurrently. Again, in Stage 2, when positive and negative stimuli of the blue-green set were reversed, the performance of the ED group was somewhat better, if anything, than that of the ID group, although the difference is insignificant $[\mathrm{F}(1 / 10)=2.65, \mathrm{p}=.13]$. Nor in Stage 3 , when the relevant dimension of the redyellow set changed, was there any indication of better ID than ED performance $[F(1 / 10)=1.13, p=.31]$. An interesting phenomenon that was noted in the previous work with goldfish is that the dimensional shift had no effect on response to the two stimuli whose signs remained the same (for example, redvertical-positive and yellow-horizontal-negative in a shift from orientation-relevant with vertical-positive to color-relevant with red-positive). It was as though the animals were dealing with the problems in terms of specific stimulus compounds rather than in terms of dimensionality. It seems, then, that sophisticated pigeons behave in these experiments like sophisticated goldfish and carp.

\section{EXPERIMENT III}

The difference between the results of Experiment II and those of Mackintosh and Little (1969) with naive pigeons in a choice situation may be due either to experience, or to method, or both. To test the hypothesis that method is the critical variable, we looked for the dimensional transfer effect in experienced animals under choice conditions.

\footnotetext{
Method

Subjects. The subjects were the 12 male homing pigeons used in Experiment II.

Apparatus. The apparatus was similar to that used in Experiment II, except that there were two pecking keys on the response panel (rather than a single key) with the feeder centered below them. All the events of the experiment were controlled automatically and registered on a six-pen event recorder.

Procedure. A noncorrection discrete-trials training procedure was employed, with a pair of stimuli presented on each trial. Response to the correct stimulus turned off both stimuli and initiated a $2-\mathrm{sec}$ reinforcement interval; response to the negative stımulus also turned of both stimuli but no food was delivered. Each session began with two free reinforcements and consisted of 40 trials with an intertrial interval of $30 \mathrm{sec}$. The animals were adapted to the new situation in four sessions in which only one of the two keys was illuminated (by white light) on each trial and response to that key was reinforced; the lighted key appeared equally often on the right and left sides in balanced orders.

In Stage 1 of the experiment, all animals were trained for the first 10 sessions with the red and yellow horizontal and
}

vertical lines which they had encountered previously. The red vertical was paired with the yellow-horizontal, and the redhorizontal was parred with the yellow-vertical Each stimulus appeared equally often on the right and on the left, and both pairs were presented equally often in each session in balanced orders. For each anımal, the posituve and negative stimul were the same as in Stage 1 of Experiment II. That is, color was relevant and orjentation irrelevant for half the animals, while for the remaining anımals orientation was relevant and color irrelevant. In the rext nine sessions (11-19), the animals were trained with the blue and green diagonals, blue $-45^{\circ}$ parred with

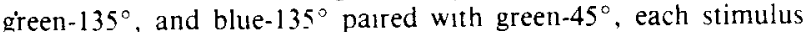
appearing equally often on right and left and each pair presented equally often in each session. For one yellow-positive, two redpostlive, one horizontal-positive, and two vertical-positive animals, color was relevant and orientation irrelevant (blue positive for three and green positive for three). For the remaining anımals, orientation was relevant and color irrelevant $\left(45^{\circ}\right.$ positive for three and $135^{\circ}$ positive for three). For half the anımals, therefore, the shift was ID, while for the rest, it was ED Attention theory, of course, predicts better performance in the ID animals.

In Stage 2, the effect of reversal was studied There were 13 sessions with the red and yellow horizontals and verticals. The stımuli were paired and presented as in Stage 1, except that red rather than yellow, or yellow rather than red, was reinforced for the color animals, and horizontal rather than vertical, or vertical rather than horizontal, was reinforced for the orientation animals. If the ID anımals came to this problem with stronger attenison in the relevant dimension, they might be expected to show more negative transfer in the earliest trials but-by the same logic as that which predicts the overlearning reversal effect (Sutherland \& Mackintosh, 1971)-more rapid mastery than the ED animals.

In Stage 3, both sets of stumuli were encountered concurrently for eight sessions. The stumul were paired as before and differentially remforced as before-the blue-green pars as in Stage 1 and the red-yellow pairs as in Stage 2. Each parr of stimul was presented equally often in each session, and spatial arrangements were balanced over parrs of sessions. The expectation here again was that any effect of dimensional incongruence in the ED anımals might be more evident when the anımals were required to deal with both sets of stmuli in the same series of trials.

In Stage 4, the effect of a double dimensional shift was studied. There were seven sessions of concurrent training with both sets of stimuli as in Stage 3. Of the six ID animals, the three for which color was relevant in both problems were shifted to orientation relevant in both problems, while the three for which orientation was relevant in both problems were shifted to color relevant in both problems. Of the six ED anmals, the three for which color was relevant in the red-yellow problem and orientation relevant in the blue-green problem were shifted to orientation relevant in the red-vellow problem and color relevant in the blue-green problem The three animals for which orientation was relevant in the red-yellow problem and color relevant in the blue-green problem were shifted to color relevant in the red-yeliow problem and onentation relevant in the blue-green problem. The prediction from attention theory is not entirely clear here, although we certainly should expect that once the chance level of performance has been exceeded the ID animals should do better than the ED anmals The former have merely to shift atiention from one dimension, relevant in both sets of stimuli, to another. The latter, which presumably have been operaung in the previous stage on the basis of a complex conditional discrumination (for example, color relevant in the red-yellow set, but orientation relevant in the blue-green set), now must reverse that discrimination 


\section{Results}

Learning curves based on percentage of correct choice in each session are plotted in Figure 3. In Stage 1, both groups rapidly achieved errorless performance in the first problem (with the redyellow set) and then in the second (with the bluegreen set). The mean error score of the ID group was slightly less than that of the ED group, but the difference is statistically unreliable $(F<1)$. Inspection of individual learning curves shows them to be of the same shape as the mean curves and gives no hint of a systematic difference between the two groups. In Stage 2 , when positive and negative stımuli in the red-yellow set were reversed, ID and ED scores were very much the same $(F<1)$, and in Stage 3, with both sets of stimuli combined, the performance of both groups was essentially errorless (curves not shown to conserve space). Nor in Stage 4, with relevant and irrelevant dimensions reversed in both sets of stimuli, was there any difference between ID and ED scores $(F<1)$. Again in these choice experiments, then, we failed to find any indication that discriminative performance is better when the same dimension is relevant in two problems than when the relevant dimension of one problem is irrelevant in the other.

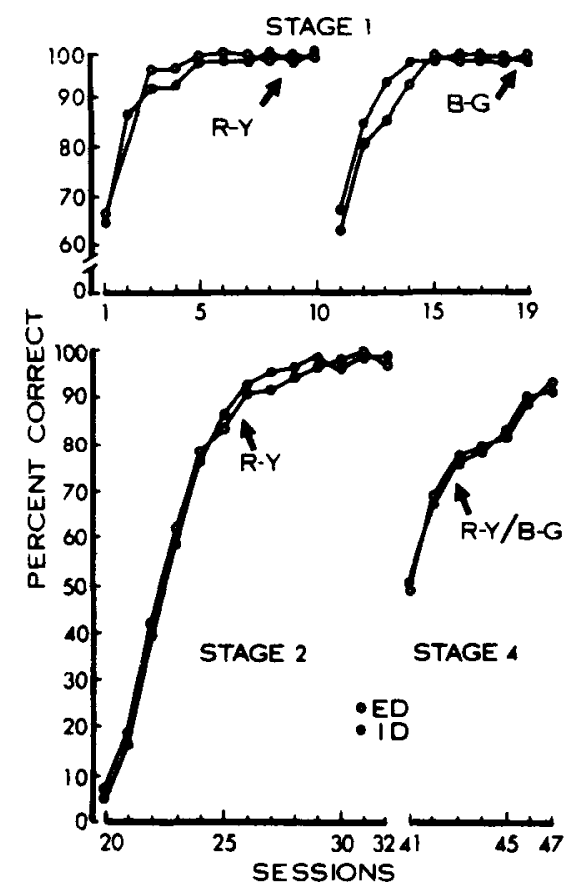

Figure 3. The performance of sophisticated pigeons under choice conditions in Experiment III. ED, extradimensional; ID, intradimensional; R-Y, red and yellow horizontals and verticals; B-G, blue and green diagonals; R-Y/B-G, both sets of stimuli combined.

\section{EXPERIMENT IV}

The foregoing results suggested that both experience and method might be important variables - that the effect appears only in naive pigeons under choice conditions - and we decided to look for it there. The experiment was patterned quite closely after that of Mackintosh and Little (1969) to begin with, except that more training was given in the original problem (which should, if anything, have increased attention in the relevant dimension, thus heightening the effect), but further tests were made after the first transfer problem.

\section{Method}

Subjects. The subjects were 12 experimentally naive homing pigeons obtained from a local breeder. They were maintained at $80 \%$ of free-feeding weight on a 24 -h feeding schedule in a ventilated and temperature-controlled laboratory room.

Apparatus. The one-key apparatus of Experiment II was used in the pretraining, and the two-key apparatus of Experiment III was used in the experiment proper.

Procedure. The animals were feeder-trained, autoshaped to peck a white key, and reinforced on a VI 30-sec schedule in the one-key apparatus. Then they were trained for six sessions in the two-key apparatus, differentially reinforced for pecking at the one key illuminated (with white light) on each trial; the position of the lighted key varied in balanced orders from trial to trial. Each of these pretraining sessions and each subsequent experimental session consisted of 40 trials with an intertrial interval of $30 \mathrm{sec}$, and each began with two free reinforcements.

In Stage 1 of the experiment, all animals were trained as in the previous experiment with the red and yellow horizontals and verticals for 20 sessions. As before, red was positive for three of the animals, yellow for three, horizontal for three, and vertical for three. In Sessions 21-26, all animals were trained as before with the blue and green diagonals. For one yellow-positive, two red-positive, one vertical-positive, and two horizontal-positive animals, color was relevant and orientation irrelevant (blue positive for three, green positive for three). For the remaining animals, orientation was relevant and color irrelevant $\left(45^{\circ}\right.$ positive for three, $135^{\circ}$ positive for three). The shift was ID for half the animals and ED for the remaining animals. In Sessions 27-29, the animals were trained as in the previous experiment with both sets of stimuli concurrently, again in the hope of maximizing any effect of dimensional incongruence.

In Stage 2, the effect of a double reversal was studied. There were 25 sessions, with the two sets of stimuli presented concurrently in each, the formerly unreinforced member of each pair now reinforced. Here, again, reversal was used as a conventional test of "analyzer" strength, which should be greater in the ID group.

In Stage 3, the effect of a dimensional shift was studied. There were nine sessions with both sets of stimuli presented concurrently, the blue-green pairs differentially reinforced as before, but the relevant and irrelevant dimensions of the redyellow pairs interchanged. Half the color-relevant animals were shifted to horizontal-positive and the rest to vertical-positive. Half the orientation-relevant animals were shifted to red-positive, and the rest to yellow-positive. With this change, the former ID animals became ED animals, and the former ED animals became ID animals; subjects and conditions were in this way unconfounded. 


\section{Results}

The performance of the two groups in each stage of the experiment is plotted in Figure 4. Trained with the red-yellow set of stimuli in the early sessions of Stage 1, the naive animals of the present experiment improved somewhat less rapidly than did the experienced animals of Experiment III, which is perhaps to be expected. Shifted to the blue-green problem, the ID group showed some transient superiority to the ED group, but the difference, as measured over all six sessions $(21-26)$, is not statistically reliable $(\mathrm{F}<1)$. Because Mackintosh and Little (1969) had found a significant difference between their groups in errors to the criterion of only $80 \%$ correct choice, we had decided in advance of our own experiment to look at the results in terms of that criterion. Analysis of variance showed a significant color-orientation difference--animals for which color was relevant made fewer errors than those for which orientation was relevant $[\mathrm{F}(1 / 8)=19.22, \mathrm{p}=.002]$-but the difference between groups (the ID-ED difference) was not significant $[\mathrm{F}(1 / 8)=2.39, \mathrm{p}=.16]$, and neither was the interaction $(\mathrm{F}<1)$.

As may be seen in Table 2 , the pattern of results in this first transfer problem was very much the same as that reported by Mackintosh and Little, and it might be thought that there was at least a

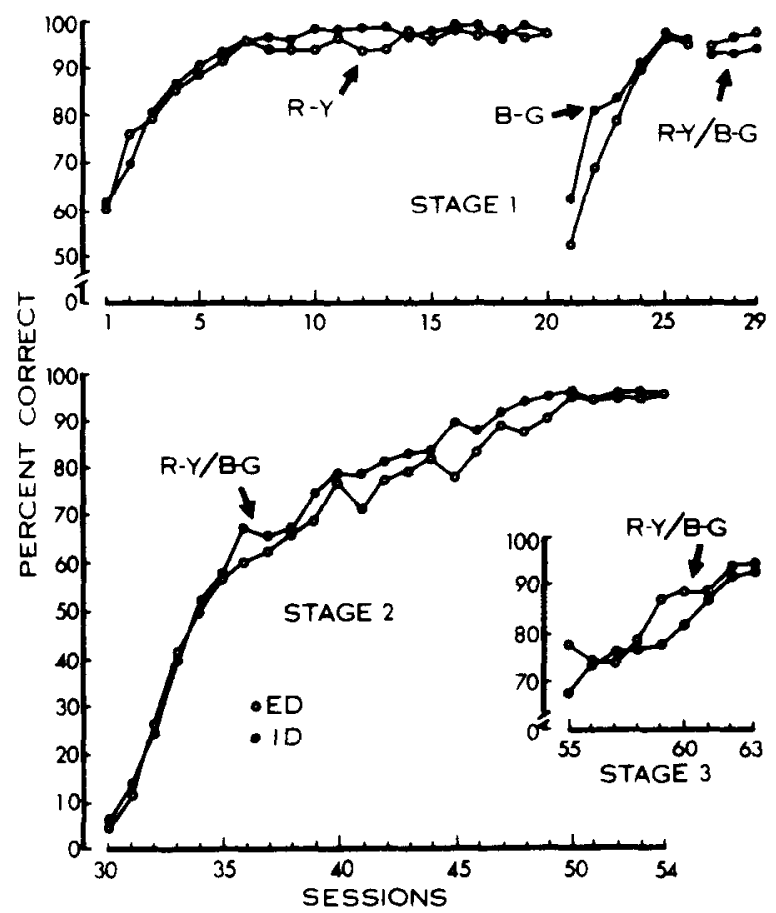

Figure 4. The performance of naive pigeons under choice conditions in Experiment IV. ED, extradimensional; ID, intradimensional; $R-Y$, red and yellow horizontals and verticals; B-G, blue and green diagonals; R-Y/B-G, both sets of stimuli combined.
Table 2

Comparison of the Results of Mackintosh and Little (1969) With Those Obtained in Experiment IV

\begin{tabular}{|c|c|c|}
\hline \multirow[b]{2}{*}{ Condition } & \multicolumn{2}{|c|}{$\begin{array}{c}\text { Mean Frrors to the } \\
80 ; \text { Cruterion }\end{array}$} \\
\hline & $M \& L$ & Exp. IV \\
\hline Color-Color & 13.5 & 13.0 \\
\hline Orientation-Color & 30.5 & 21.3 \\
\hline Orientation-Orientation & 54.0 & 43.0 \\
\hline Color-Orientation & 67.0 & 58.3 \\
\hline
\end{tabular}

real tendency toward a dimensional transfer effect which might have reached statistical significance with larger samples or more reliable measures, but inspection of individual records suggests another interpretation. The ID-ED difference for animals trained with color as the relevant dimension was due primarily to the poor performance in the first session of one bluepositive ED animal with a very strong initial preference for green, which it should not have displayed according to attention theory if it had come to the problem with a set for orientation (the previously relevant dimension). The rate of learning in this animal was, in fact, higher by far than in any other (its probability of correct choice being $95 \%$ in the second session), which again would not be expected from attention theory on the assumption that the animal came to the problem with strong attention to orientation. The ID-ED difference for animals trained with orientation as the relevant dimension was due for the most part to rapid acquisition by one of the ID animals, although the individual curves for the two groups of animals did seem to differ consistently in shape, the ID curves being negatively accelerated throughout while the ED curves showed some initial positive acceleration. In the second session, for example, all of the ED (orientation) animals continued to perform at the $50 \%$ level, while all of the ID animals already gave evidence of considerable progress. Even if this statistically unevaluatable difference is taken seriously, however, it cannot safely be regarded as an attentional effect without a control for general transfer. More rapid improvement in the ID animals trained with orientation relevant might be expected also from the fact that their first problem (orientation) was substantially more difficult than the first problem of the ED animals (color). (It is interesting to note, in this connection, that the relative difficulty of the orientation problems was much greater in the choice situation than in the single-stimulus freeoperant situation, where the color and orientation problems were about equal in difficulty.)

Whatever the meaning of the ID-ED difference, it certainly was highly transient, disappearing entirely 
in Sessions 24-26. In Sessions 27-29, when the animals were trained concurrently with both sets of stimuli, ED performance was better, if anything, than ID performance, although not significantly so $(F<1)$. Nor did the ID-ED difference approach significance either in Stage 2 (double reversal) or in Stage 3 (when a dimensional shift in the red-yellow set exchanged groups and conditions).

\section{DISCUSSION}

These experiments were predicated on the assumption that, at least under certain conditions, pigeons show better intradimensional than extradimensional transfer (Mackintosh \& Little, 1969). They were designed to check on the possibility that a like effect had not been found in goldfish (Tennant \& Bitterman, 1973) and carp (Tennant \& Bitterman, 1975), either because the fishes used were experienced (rather than naive, as were Mackintosh and Little's pigeons) or because the fishes were trained under free-operant single-stimulus conditions (rather than discrete-trials choice conditions, as were the pigeons). The negative results of the first experiment (in which naive goldfish were trained under singlestimulus conditions) suggested that the difference was not due to experience alone. The negative results of the second experiment (in which sophisticated pigeons were trained under single-stimulus conditions) showed that the difference was not due simply to the species of animals used, and the negative results of the third experiment (in which sophisticated pigeons were trained under choice conditions) showed that the difference was not due to method alone. The results of the fourth experiment (in which naive pigeons were trained under choice conditions) raise the question of whether pigeons do, in fact, show greater intradimensional than extradimensional transfer under any conditions at all.

A puzzling feature of Mackintosh and Little's results, in view of the high asymptotic level of performance achieved by pigeons in our own choice experiments (see Figures 3 and 4 ), is the modest criterion employed $(80 \%$ correct). If the asymptotic performance of their animals was in fact higher, it would be important to know why so modest a criterion was selected. If the asymptotic performance of their animals was in fact poor, the results lose something of their impact. In a recent experiment with chickens trained in a series of three (choice) problems, Hamlin (1975) found what might be taken to be better ID than ED transfer from the first problem to the second-the discrimination of triangles was better after training with stripes than after training with colors-but the experiment was unbalanced in design. In the third problem, stripe discrimination was the same whether the animals had been trained with triangles or with colors in the second. There is, then, no clear evidence of the dimensional transfer effect in birds. If there is indeed such an effect, it is small in magnitude, highly transient, and occurs under a range of conditions much too restricted to be explained in terms of conventional attention theory.

\section{REFERENCES}

Bitterman, M. E. Review of Sutherland and Mackintosh. Mechanisms of animal discrimination learning. American Journal of Psychology, 1972, 85, 301-303.

Hamlin. P. H. Observing responses as an index of attention in chickens. Joumal of Experimental Psychology Animal Behavtor Processes, 1975, 1, 221-234.

Mackintosh, N. J., \& Little. L. Intradimensional and extradimensional shitt learning in pigeons. Psychonomic Scrence. 1969, 14, 5-6.

Shepp. B. E. \& Eimas. P. D. Intradimensional and extradimensional shitts in the rat. Journal of Comparative and Physological Psychology. 1964. 57, 357-361.

Shepp, B. E.. \& Schrier, A. M. Consecutive intradimensional and extradimensional shitts in monkeys. Journal of Comparative and Physiological Psychology. 1969, 67. 190-203.

Sutherland, N. S..\& MaCkintosh. N. J. Mechantsms of animal discrimmaton leaming. New York: Academic Press. 1971.

Tennant. W. A.. \& Bitterman. M. E. Some comparisons of intra- and extradimensional transfer in discriminative learnmg of goldtish. Joumal of Comparateve and Phisiological Psrchology: 1973.83, 134-139.

Tennant, W. A., \& Bitterman, M. E. Blocking and overshadowing in two species of fish. Joumal of Experimental Psychology. Animal Behavior Processes, 1975, 1, 22-29.

Woodard. W. T.. \& BitTerman. M. E. Improved techniques for the measurement of consummatory behavior in fishes. Behavior Reseurch Mcthods \& Instrumentation. 1974. 6. 323-324.

(Recerred for publication November 3. 1975 ; accepted November 20, 1975.) 